\title{
Variabilidade mensal e horária de elementos meteorológicos na área experimental do Projeto ESECAFLOR na Floresta Nacional de Caxiuanã, Pará, Brasil \\ Monthly and hourly variability of meteorological elements in the ESECAFLOR Project Experimental area of the Caxiuanã National Forest in Pará, Brazil
}

\author{
Antonio Carlos Lôla da Costa', João de Athaydes Silva Júniorl', Alex Antonio Ribeiro de Oliveira", \\ Carlos Leandro Ribeiro da Costa", Lucy Miranda Rowland ${ }^{\mathrm{III}}$, Patrick Meir"II, Alan Cavalcanti da Cunha ${ }^{\mathrm{IV}}$, \\ Yadvinder Malhì', Hernani José Brazão Rodrigues' \\ "Universidade Federal do Pará. Belém, Pará, Brasil | "Museu Paraense Emílio Goeldi/MCTIC. Belém, Pará, Brasil \\ '"University of Edinburgh. Edinburgh, Reino Unido | 'Vuniversidade Federal do Amapá. Macapá, Amapá, Brasil
}

vUniversity of Oxford. Oxford, Reino Unido

\begin{abstract}
Resumo: Florestas tropicais são reguladoras do clima e o padrão de variabilidade dos elementos meteorológicos representa fisicamente interdependência entre os fluxos térmicos e hídricos para a floresta e a atmosfera. A presente investigação analisa uma série de dados (precipitação, temperatura do ar, umidade relativa do ar, radiação solar global, velocidade e direção do vento) na Floresta Nacional de Caxiuanã, Pará. O estudo ocorreu entre 2005 e 2013, usando-se uma classificação sazonal, definida da seguinte forma: dezembro a junho, como chuvoso, e julho a novembro, como menos chuvoso. Dois métodos estatísticos básicos foram aplicados para testar as diferenças sazonais e avaliar a "sincronicidade" entre as variações sazonais dos elementos meteorológicos: a) teste não-paramétrico (Mann-Whitney; p <0,05) para a análise horária; b) Teste de estatística circular (método Watson-Williams). Concluímos que em termos horários a direção do vento e a temperatura do ar mudaram entre períodos úmidos e secos, mas a intensidade do vento não mostrou alterações significativas. Também houve mudanças significativas na radiação global, precipitação e umidade relativa. A variabilidade da velocidade do vento foi sincronizada com variabilidade na direção do vento, precipitaçãa e temperatura do ar, mas não com a umidade relativa e radiação solar total. Esses resultados geram uma descrição básica dos mecanismos físicos que controlam a dinâmica do tempo sazonal nas florestas tropicais.
\end{abstract}

Palavras-chave: Série histórica. Sazonalidade. Análise estatística.

Abstract: Tropical forests play an important role in regulating the climate. Variability in the patterns of the main meteorological elements are controlled by the interdependence of the flows of heat and water between the forest and the atmosphere. This research analyzes a range of meteorological data from the Caxiuanã National Forest, Pará, Brazil, from 2005 to 2013, including: rainfall, air temperature, relative humidity, global solar radiation, wind speed, and wind direction. Seasons were classified into wet season (December to June) and dry season (July to November). Two basic statistical methods were applied to test for differences by season and to assess the 'synchronicity' between seasonal variations of meteorological elements: a) non-parametric test (Mann-Whitney; p < 0.05) for hourly analysis, b) a circular statistics test (WatsonWilliams method). We conclude that hourly wind direction and air temperature changed between wet and dry periods but wind intensity showed no significant change. There were also significant changes to the global radiation, rainfall and relative humidity. The variability of wind speed was synchronized with variability in wind direction, rainfall and the air temperature, but not with relative humidity and total solar radiation. These results generate a basic description of the physical mechanisms which control seasonal weather dynamics in tropical forests.

Keywords: Historic Series. Seasonality. Statistical analysis.

COSTA, A. C. L., J. A. SILVA JÚNIOR, A. A. R. OliVEIRA, C. L. R. COSTA, L. M. ROWLAND, P. MEIR, A. C. CUNHA, Y. MALHI \& H. J. B. RODRIGUES, 2017. Variabilidade mensal e horária de elementos meteorológicos na área experimental do Projeto ESECAFLOR na Floresta Nacional de Caxiuanã, Pará, Brasil. Boletim do Museu Paraense Emílio Goeldi. Ciências Naturais 11(3): $365-375$. Autor de correspondência: Antonio Carlos Lôla da Costa. Universidade Federal do Pará. Instituto de Geociências. Faculdade de Meteorologia. Rua Augusto Corrêa, 1 - Guamá. Belém, PA, Brasil. CEP 66075-110 (lola@ufpa.br).

Recebido em 26/01/2015

Aprovado em 06/03/2017

Responsabilidade editorial: Maria de Lourdes Pinheiro Ruivo e Rogério Rosa da Silva

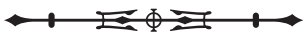




\section{INTRODUÇÃO}

A região amazônica ocupa uma área aproximada de $5,5 \times 10^{6} \mathrm{~km}^{2}$ e vem despertando grande interesse em pesquisadores do mundo inteiro, devido às elevadas taxas de desmatamento e à consequente ameaça de extinção de espécies (Costa et al., 2010; Marengo \& Espinoza, 2016). Nas últimas décadas, as pesquisas feitas na floresta amazônica têm dado ênfase à ampliação do conhecimento sobre os processos físicos, fisiológicos e biológicos, em interação com o sistema solo-planta-atmosfera (Doughty et al., 2015), o quais são indicadores que representam as alterações ou perturbações referentes às relações entre a vegetação e o clima da floresta.

Os elementos meteorológicos são condicionantes climáticas que, invariavelmente, constituem-se em gradientes abióticos, sendo utilizados como variáveis independentemente de avaliação de cenários futuros sobre a dinâmica ecossistêmica dos diferentes biomas em todo o globo, onde esses elementos condicionam a sobrevivência e a adaptação de diversas espécies de plantas e de animais (Souza et al., 2014). Por exemplo, em 2005 e 2010, a bacia amazônica experimentou duas fortes secas, provocadas por mudanças no regime hidrológico tropical, potencialmente associado com mudanças climáticas globais, tais como previstas por alguns modelos ambientais (Doughty et al., 2015).

Souza et al. (2014) descrevem que uma das questões mais importantes de avaliação de cenários futuros de longo prazo para o clima está justamente relacionada às incertezas nas formulações mais completas dos processos físicos, químicos e biológicos, incluindo as complexas interações clima-vegetação de projeções climáticas e ambientais.

Além das incertezas sobre os cenários de emissões (dependentes da socioeconomia, da demografia, da composição das fontes de geração de energia, de atividades de uso da terra), há aquelas referentes ao padrão da variabilidade natural do sistema climático, nas quais os processos físicos e químicos da atmosfera global são 'caóticos', sensivelmente dependentes das mínimas mudanças (normalmente não lineares), com difíceis possibilidades de mensuração. Isso vale tanto para os dados observacionais quanto para os resultados dos modelos.

A atmosfera desta modalidade da floresta amazônica apresenta-se com elevadas temperaturas do $\operatorname{ar}\left(22^{\circ} \mathrm{C} \leq\right.$ $\mathrm{T} \leq 28^{\circ} \mathrm{C}$ ) e precipitações anuais que, ao longo do ano, podem apresentar grandes amplitudes $(2.300 \mathrm{~mm} \leq$ $\mathrm{P} \leq 3.500 \mathrm{~mm}$ ) (Costa et al., 2010; Fisch et al., 1996). Entretanto, estes elementos meteorológicos são bem definidos e utilizados para classificar dois períodos sazonais bem distintos: uma estação chuvosa, compreendida entre os meses de dezembro a junho, e outra menos chuvosa, que se estende de julho a novembro. Essas características estão relacionadas à grande quantidade de radiação solar que chega na região tropical e à presença da Zona de Convergência Intertropical (ZCIT), o principal sistema produtor de precipitação em nossa região, onde a ZCIT se desloca acompanhando o movimento contínuo do Sol entre as latitudes de $27,45^{\circ} \mathrm{N}$ e $27,45^{\circ} \mathrm{S}$ (Vilani et al., 2006; Costa et al., 2009).

Assim, a FLONA de Caxiuanã pode ser definida como uma floresta tropical chuvosa típica, sem perturbações antrópicas, onde se desenvolvem atualmente vários projetos de pesquisa, sobre os mais diversos temas. O interesse por pesquisas de longa duração no bioma amazônico tem crescido em decorrência da pressão antrópica que vem ocorrendo nessa região, afetando diretamente a biodiversidade deste bioma, associada às mudanças climáticas (Tabarelli et al., 2013).

Na Amazônia, experimentos de longa duração, denominados de Projetos Ecológicos de Longa Duração (PELD), são relativamente raros, mas relevantes, pois vinculam informações sobre a dinâmica do clima e as suas possíveis influências sobre estes ecossistemas tropicais e sobre a sua rica biodiversidade associada (Doughty et al., 2015; Costa et al., 2010).

Séries históricas longas de dados climáticos são extremamente importantes, porque servem como parâmetros ou gradientes físicos associados aos

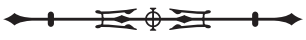


indicadores bióticos e porque suas variações interferem diretamente na dinâmica ecossistêmica da floresta, principalmente a temperatura do ar, a radiação solar global e a precipitação pluviométrica.

As séries de informações aqui utilizadas são inéditas para estes ambientes, possibilitando uma análise mais profunda sobre as variações sazonais médias horárias e mensais dos elementos meteorológicos na FLONA de Caxiuanã, em Melgaço, Pará, Brasil. A principal hipótese da pesquisa é de que todos os elementos meteorológicos estudados apresentam significativa variação sazonal, tanto na escala mensal quanto na horária.

A presente pesquisa tem como objetivo quantificar as variações mensais e horárias de elementos meteorológicos em ecossistema florestal, no período de 2005 a 2013. Estas séries têm a finalidade de gerar bases de conhecimento sobre a dinâmica dos elementos meteorológicos relevantes, como temperatura do ar, umidade relativa do ar, radiação solar global, precipitação pluviométrica, direção e velocidade do vento, de modo contínuo e em intervalos horários, de forma confiável, para subsidiar análises de cenários e de comportamentos relacionados com processos hidroclimáticos regionais, ainda pouco estudados (Molion, 1987; Souza et al., 2014).

\section{MATERIAL E MÉTODOS}

\section{LOCAL DE ESTUDO}

$O$ presente estudo foi desenvolvido na FLONA de Caxiuanã, localizada no município de Melgaço, estado do Pará, no leste da Amazônia (01 42' 30" S; 510 31' 45" O; $62 \mathrm{~m}$ de altitude). A FLONA de Caxiuanã é uma fração representativa de ecossistema tropical, resiliente, apresentando uma área de 330.000 hectares, sendo composta predominantemente por floresta de terra firme, com dossel médio entre 30 e 40 m de altura, onde a floresta cobre $85 \%$ dessa área. A diversidade varia entre 150 a 160 espécies ha-1 ${ }^{-1}$, enquanto que a densidade de indivíduos apresenta um valor médio entre 450 a 550 árvores ha ${ }^{-1}$, sendo que a sua biomassa aérea média é de $200 \mathrm{~m}^{-3}$. ha-1 (Lisboa et al., 1997). Esta floresta apresenta diversidade considerável, com espécies importantes, como Dinizia excelsa Ducke (angelimvermelho), Marmaroxylon racemosum (Ducke) Killip ex Record (angelim-rajado), Couratari guianensis Aubl. (tauari), Buchenavia grandis Ducke (tanimbuca), Swartzia racemosa Benth. (pitaíca), Dipteryx odorata (Aubl.) Willd. (cumaru), entre outras (Almeida et al., 1993).

Quanto à climatologia do local, a temperatura média anual do ar é de $25,7 \pm 0,8^{\circ} \mathrm{C}$ e a precipitação média anual é de $2.272 \pm 193 \mathrm{~mm}$. A média anual da umidade relativa do ar é de $82,3 \%$, sendo a média anual da radiação solar global de $286,1 \mathrm{~W} \cdot \mathrm{m}^{-2}$, atingindo os maiores valores no mês de julho e menores valores no mês de abril. $O$ brilho solar anual supera 2.100 horas. A direção predominante do vento é no quadrante $\mathrm{N}-\mathrm{E}$. Pela classificação climática de Köppen, o clima é do tipo 'Am' tropical quente e úmido, e subtipo climático com uma curta estação seca (Moraes et al., 1997; Silva Júnior et al., 2013; Rowland et al., 2015).

\section{DADOS UTILIZADOS}

Os dados meteorológicos utilizados neste trabalho foram obtidos através de uma estação meteorológica automática da Campbell Scientific, com datalogger modelo CR1000, localizada no topo de uma torre micrometeorológica, com 40 m de altura. O período de estudo foi de 2005 a 2013, sendo as informações armazenadas em intervalos de 30 minutos. Os elementos meteorológicos estudados foram: temperatura do $\operatorname{ar}\left({ }^{\circ} \mathrm{C}\right)$, umidade relativa do ar (\%), precipitação pluviométrica $(\mathrm{mm})$, radiação solar global $\left(\mathrm{W} \cdot \mathrm{m}^{-2}\right)$, velocidade do vento $\left(\mathrm{m} \cdot \mathrm{s}^{-1}\right)$ e direção do vento $\left({ }^{\circ}\right)$. Foram considerados dois períodos distintos: o chuvoso, no qual a precipitação é superior à evapotranspiração potencial, abrangendo os meses de dezembro a junho, e o período menos chuvoso, no qual a precipitação é inferior à evapotranspiração potencial, estendendo-se de julho a novembro. A temperatura e 
a umidade relativa do ar foram medidas através de um psicrômetro do tipo HMP45C, cuja temperatura de operação oscila entre -40 até $60{ }^{\circ} \mathrm{C}$. Foi utilizado um anemômetro modelo 03001-5 Wind Sentry, para as medidas de direção e velocidade do vento. A precipitação pluviométrica foi medida por meio de um pluviógrafo do tipo CSI, modelo CS700-L Rain Gauge, com 200 mm de diâmetro de funil e capacidade 'basculante' de até $100 \mathrm{~mm}$. h-1. A radiação solar global foi medida através de um sensor de brilho solar Sunshine Sensor, modelo BF4. Para a aquisição destas informações, foram utilizados dois dataloggers da Campbell Scientific, sendo um de modelo CR10X (2005 a 2007) e outro de modelo CR1000 (2008 a 2013).

\section{ANÁLISES ESTATÍ́STICAS}

O conjunto de dados meteorológicos gerou basicamente duas planilhas, que permitiram a análise conjunta das variáveis meteorológicas: a) a primeira para avaliar a variação mensal dos elementos meteorológicos (sazonalchuvoso e menos chuvoso); b) a segunda para avaliar a variação horária dos elementos meteorológicos, considerando-se também os dois períodos, chuvoso e menos chuvoso.

Inicialmente, realizou-se um tratamento estatístico básico, como uma avaliação descritiva dos parâmetros e, consequentemente, uma avaliação adicional para testar a normalidade das distribuições de cada um dos elementos, verificando-se se as mesmas são ou não normais.

As comparações entre as séries mensais e horárias dos elementos meteorológicos foram feitas com base em testes de hipóteses do tipo Mann-Whitney (teste $U$ de Wilcoxon-Mann-Whitney) (Ayres et al., 2005). Este teste é destinado a comparar duas amostras independentes, cujos scores tenham sido medidos pelo menos em nível ordinal. Em síntese, consideramos o seguinte: $\mathrm{H}_{\circ}=$ existência de diferenças entre os elementos meteorológicos, independentes dos períodos sazonais; $\mathrm{e}_{1}=$ as diferenças entre os elementos meteorológicos estão relacionadas com os períodos sazoais. $O$ teste $U$ foi considerado estatisticamente significativo quando $p<0,05$.

Do mesmo modo, aplicou-se o método de Rayleigh, utilizado para verificar se uma população ou amostra está distribuída uniformemente em torno de uma circunferência (Ayres et al., 2005). No presente caso, utilizou-se como parâmetro básico a direção do vento, no qual a hipótese nula $\left(\mathrm{H}_{\mathrm{o}}\right)$ declarou que os elementos meteorológicos apresentaram distribuição circular uniforme em torno da direção do vento. A hipótese alternativa $\left(H_{1}\right)$, por sua vez, afirma que os elementos meteorológicos não são uniformemente distribuídos em torno da circunferência na referida FLONA de Caxiuanã (método Watson-Williams). Portanto, se for o caso, provavelmente existe uma direção predominante do vento entre os dois períodos sazonais, chuvoso e menos chuvoso, à qual é também possível correlacionar ou sincronizar outras variáveis meteorológicas.

Admitiu-se que, se o p-valor para o teste estatístico circular obtido fosse menor que o nível de significância (alfa $<0,05$ ), rejeitar-se-ia a hipótese e concluir-se-ia que há uma orientação direcional do vento na FLONA de Caxiuanã, sendo esta representada pela média angular da amostra.

O método de Rayleight (teste $V$ ) é usado para testar um valor paramétrico de média angular contra a hipótese de uniformidade em torno da circunferência, onde $\mathrm{H}_{\text {。 }}$ afirma que a população dos dados é uniforme ao redor da circunferência e $H_{1}$ afirma que esta população não é uniformemente distribuída ao redor da circunferência (Ayres et al., 2005). É relevante frisar, contudo, que se a $\mathrm{H}_{\text {。 }}$ é aceita, não se deve descartar que a possibilidade de outra média angular, diferente do valor esperado, de fato exista.

\section{RESULTADOS E DISCUSSÕES}

\section{VARIABILIDADE MÉDIA MENSAL DOS ELEMENTOS METEOROLÓGICOS}

A variabilidade média mensal da temperatura do ar, da umidade relativa do ar, da radiação solar global, da 
precipitação e da velocidade do vento é apresentada na Figura 1. A temperatura média anual do ar foi de $25,9^{\circ} \mathrm{C}$, tendo os maiores valores médios ocorridos nos meses de agosto a novembro, no período menos chuvoso da região, enquanto os menores valores foram observados nos meses de janeiro a abril, no período mais chuvoso. A temperatura média anual apresentou desvio padrão de $0,6^{\circ} \mathrm{C}$ e coeficiente de variação de $2,4 \%$, indicando pouca variabilidade deste elemento meteorológico. Estes resultados são semelhantes aos apresentados por Braga et al. (2005).

A baixa variabilidade da temperatura do ar está associada com a localização geográfica da FLONA de Caxiuanã, onde o sol apresenta uma declinação máxima em torno de $23,45^{\circ}$ Norte e Sul, fato que proporciona significativa disponibilidade energética durante todo o ano, implicando baixa variabilidade mensal deste elemento meteorológico neste local. $\bigcirc$ resultado do teste $U$ foi significativo $(p=0,0045)$, demonstrando que ocorreram diferenças relevantes entre o padrão de variação da temperatura média mensal em ambos os períodos sazonais (chuvoso e menos chuvoso).

Como esperado, a umidade relativa do ar apresentou padrão de variação inverso ao evidenciado pela temperatura do ar. Embora a umidade seja elevada durante todo o ano, os maiores valores foram observados durante o período mais chuvoso da região. O valor médio anual da umidade relativa do ar foi de $82 \%$, com desvio padrão de 5,3\% e coeficiente de variação de 6,4\%, indicando também pequena variabilidade média anual deste elemento meteorológico.

O elevado valor anual da umidade relativa do ar está diretamente associado com a abundante disponibilidade de energia e com a hídrica da região, aspectos que proporcionam elevada taxa de evapotranspiração emitida pela vegetação para a atmosfera (Gat \& Matsui, 1991). O teste $U$ foi utilizado para verificar a existência de diferenças significativas entre a variação da umidade relativa média mensal do ar em ambos os períodos, resultando em $p=0,0045$ (significativo). Houve, portanto, diferenças

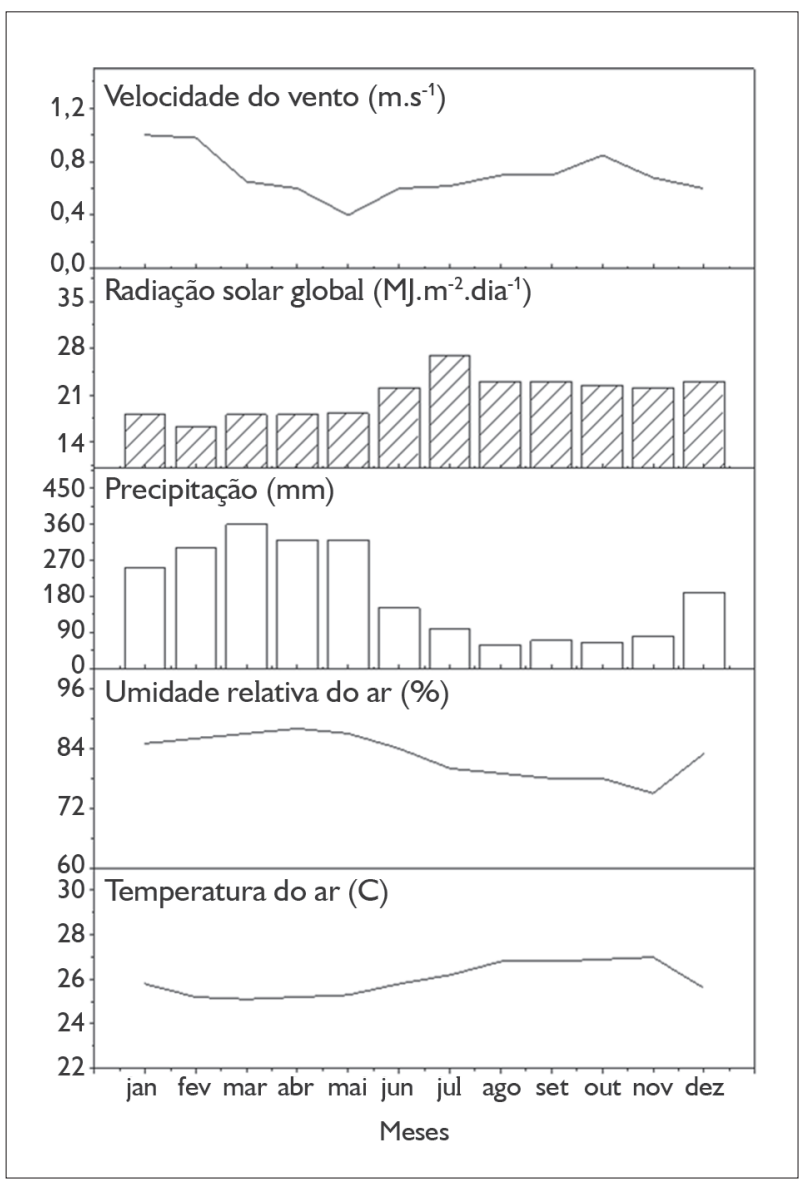

Figura 1. Variabilidade média mensal da temperatura do ar, umidade relativa do ar, radiação solar global, precipitação e velocidade do vento na FLONA de Caxiuanã, no período de 2005 a 2013.

significativas entre o padrão de umidade relativa do ar média mensal nos períodos chuvoso e seco.

Em relação à radiação solar global, foi observada considerável sazonalidade, sendo que os maiores valores médios ocorreram no mês de julho, com 27,6 MJ.m². dia $^{-1}$, enquanto os menores foram registrados no mês de fevereiro, com apenas 16,2 MJ.m². dia-1. Os valores máximos absolutos observados nos períodos seco e chuvoso foram, respectivamente, de 47,2 e 46,9 MJ.m². $\mathrm{dia}^{-1}$. A radiação solar global média diária apresentou uma redução de 12\% no seu valor durante o período chuvoso, quando comparado com o menos chuvoso. O valor médio anual foi de $20,6 \mathrm{MJ} \cdot \mathrm{m}^{-2} \cdot \mathrm{dia}^{-1}$. 
A ampla variabilidade mensal da radiação solar global está associada, principalmente, com a nebulosidade da região, que é regulada pela zona de convergência intertropical, principal sistema meteorológico regulador do tempo durante o período chuvoso desta região. Durante o período menos chuvoso, atuam também os sistemas convectivos e as zonas de instabilidade, entre outros (referência). $O$ teste $U$ foi utilizado para verificar se há diferenças significativas entre a variação da radiação solar global média mensal em ambos os períodos, resultando em $p=0,0045$ (significativo). Houve, portanto, diferenças significativas entre o padrão de umidade relativa do ar média mensal nos períodos chuvoso e seco.

A precipitação média mensal na FLONA de Caxiuanã foi o elemento meteorológico a apresentar a maior variabilidade, sendo os mais expressivos valores observados no mês de março, enquanto os menos expressivos ocorreram durante o mês de agosto. Há nítida sazonalidade da precipitação na FLONA de Caxiuanã, representando o período chuvoso entre os meses de dezembro a junho, com um total médio precipitado de $1.717,3 \mathrm{~mm}$, isto é, $76,3 \%$ do total anual. Durante o período menos chuvoso, que se estende entre os meses de julho a novembro, o total médio precipitado foi de 534 mm, o que corresponde a $23,7 \%$ do total anual.

Estudos realizados por Oliveira et al. (2008) também evidenciaram esta sazonalidade regional. Na média climatológica da região, os períodos chuvoso e menos chuvoso representam, respectivamente, 72,5 e 27,5\% do total precipitado anualmente. As precipitações durante o período menos chuvoso foram causadas, principalmente, por sistemas convectivos e por linhas de instabilidade, enquanto que, no período chuvoso, as precipitações foram causadas por sistemas de grande escala atuantes na região, como a Zona de Convergência Intertropical (Molion, 1987; Fisch et al., 1998). No período de 2005 a 2013, a precipitação média anual foi de $2.106,9 \mathrm{~mm}$. 0 maior valor precipitado em um mês foi registrado no mês de março de 2008, com 592,8 mm, enquanto o menor valor registrado foi em outubro de 2010, com apenas 11,8 $\mathrm{mm}$. $\bigcirc$ teste $U$ foi utilizado para verificar a existência de diferenças significativas entre a variação da precipitação média mensal em ambos os períodos, resultando em $p<0,05$ (significativo). Houve, portanto, diferenças significativas entre o padrão de precipitação média mensal nos períodos chuvoso e seco.

A velocidade média mensal do vento apresentou baixo valor durante todos os meses do ano, mas os maiores valores absolutos foram registrados durante o período chuvoso da região. Destaque ocorreu durante os meses de janeiro e fevereiro, que apresentaram valores médios superiores a 1,0 m.s. ventos provenientes de sistemas de grande escala (Zona de Convergência Intertropical), muito frequentes durante este período na região. $O$ valor médio anual da velocidade do vento foi de $0,86 \mathrm{~m} \cdot \mathrm{s}^{-1}$, com desvio padrão de $0,2 \mathrm{~m} \cdot \mathrm{s}^{-1}$ e coeficiente de variação de 14,3\%.

A variabilidade mensal está diretamente associada ao aquecimento diferencial da superfície, que causa diferentes pressões atmosféricas e, consequentemente, diferentes precipitações nesta região. $O$ teste $U$ foi utilizado a fim de verificar a existência de diferenças significativas entre a variação da velocidade média mensal do vento em ambos os períodos, resultando em $p=0,5698$ (não significativo). Não houve, assim, diferenças significativas do padrão de variação da velocidade média mensal para os períodos chuvoso e seco.

\section{VARIABILIDADE MÉDIA HORÁRIA DOS ELEMENTOS METEOROLÓGICOS}

Estatisticamente, em relação às medidas horárias, todas as cinco variáveis não apresentaram distribuição normal em suas respectivas frequências ao longo do período de estudo. Então, para comparar a variação entre os elementos meteorológicos no que se refere aos períodos chuvoso e seco, utilizou-se o teste estatístico não paramétrico $U$ (Mann-Withney), com significância $p$ $<0,05$. $O$ teste avaliou diferenças significativas entre as 
medidas dos elementos meteorológicos nos períodos chuvoso e menos chuvoso, computados a partir de janeiro de 2005 a dezembro de 2013.

A variação sazonal média horária da temperatura do ar, da umidade relativa do ar, da radiação solar global, da precipitação pluviométrica e da velocidade do vento foi apresentada na Figura 2. Os maiores valores médios de temperatura do ar ocorreram às 13 horas, nos dois períodos analisados. Ressalta-se que a temperatura média horária durante o período menos chuvoso apresentou valores maiores do que durante o período chuvoso, sendo que, entre 4 e 7 horas, eles apresentaram-se muito próximos em ambos os períodos. Resultados semelhantes foram registrados nos estudos desenvolvidos por Costa et al. (2006b) na FLONA de Caxiuanã. A temperatura média do ar durante o período chuvoso apresentou valor médio de $25,1^{\circ} \mathrm{C}$, enquanto, no período menos chuvoso, este valor foi de $26,2{ }^{\circ} \mathrm{C}$.

Como a umidade relativa do ar varia inversamente com a temperatura do ar, esta última apresentou os menores valores por volta das 14 horas local, e os maiores valores durante todos os horários noturnos. O valor médio deste elemento meteorológico durante os períodos chuvoso e seco foram, respectivamente, de $93,3 \%$ e $88,1 \%$, evidenciando valores elevados durante todas as horas do dia. Nas primeiras horas do dia, os valores são muito próximos, independente da época do ano. As pequenas diferenças aparecem em horários diurnos e nos primeiros horários noturnos. As amplitudes diárias durante os períodos seco e chuvoso foram de $19,6 \%$ e $11,1 \%$, respectivamente.

O valor máximo diário da radiação solar global durante o período seco foi de $749,9 \mathrm{~W} \cdot \mathrm{m}^{-2}$, enquanto que durante o período chuvoso este valor foi de $615,1 \mathrm{~W} . \mathrm{m}^{-2}$. Os valores médios diários observados durante o período seco foi de $438,5 \mathrm{~W} \cdot \mathrm{m}^{-2}$, ao passo que durante o período chuvoso este valor foi de $340,8 \mathrm{~W} \cdot \mathrm{m}^{-2}$. Durante o período chuvoso, a radiação solar difusa superou a radiação solar direta, em grande parte do dia. Os valores máximos e médios da radiação solar difusa foram de 343,0 W.m² e

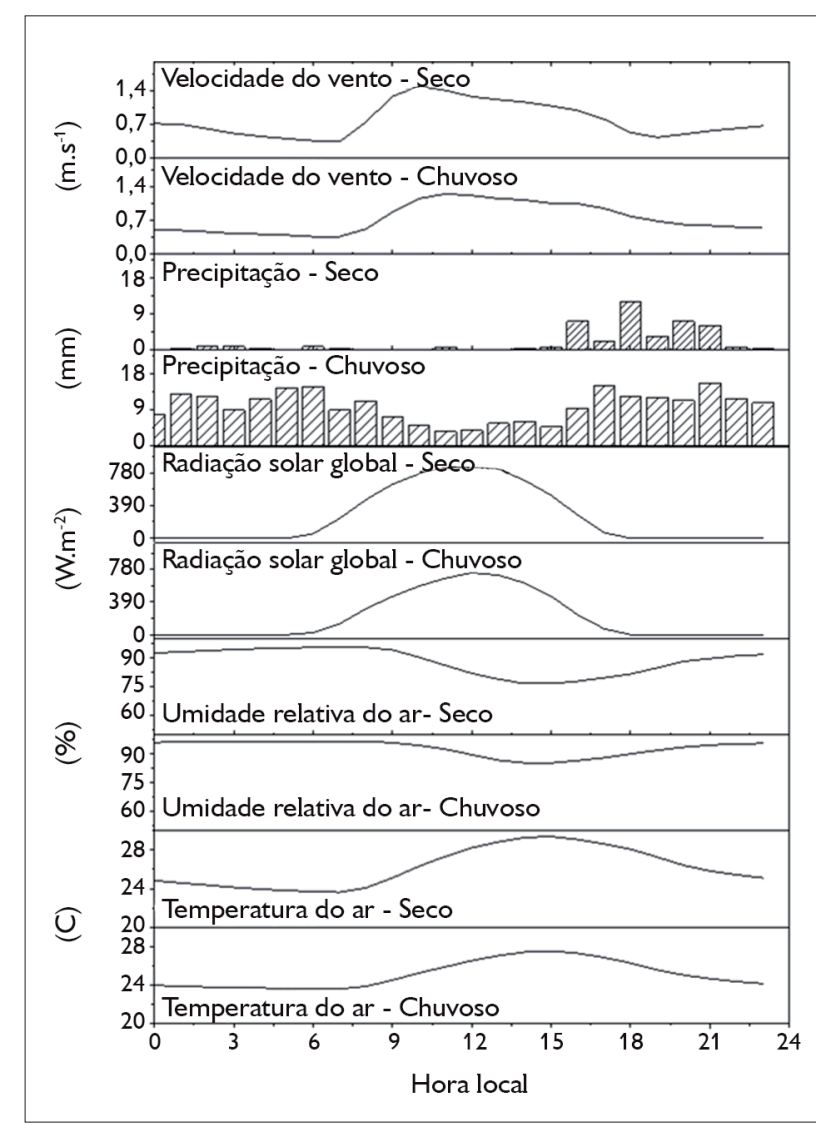

Figura 2. Variabilidade média horária da temperatura do ar, umidade relativa do ar, radiação solar global, precipitação e velocidade do vento na FLONA de Caxiuanã, no período de 2005 a 2013.

de 193,3 W. $m^{-2}$, respectivamente. A radiação solar direta apresentou valores máximos e médios de $304,1 \mathrm{~W} \cdot \mathrm{m}^{-2} \mathrm{e}$ de $147,0 \mathrm{~W} \cdot \mathrm{m}^{-2}$, respectivamente.

Durante o período seco, a radiação solar direta superou a radiação solar difusa durante todo o dia, com valores máximos e médios diários de 481,9 W.m-2 e de 265,6 W. $\mathrm{m}^{-2}$, respectivamente. Em relação à radiação solar difusa, foram verificados valores máximos e médios diários de 273,3 W.m-2 e de 160,4 W.m-2 , respectivamente. Estes resultados estão mais diretamente associados com a variabilidade anual da nebulosidade nesta região.

As precipitações médias horárias durante o período chuvoso da região foram maiores no final da tarde, ocorrendo ao longo de todo o dia. Durante o período

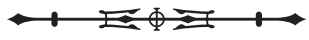


seco da região, as precipitações foram registradas, preferencialmente, no final da tarde e durante as horas noturnas, caracterizando um regime de precipitações convectivas. As maiores precipitações registradas no intervalo de uma hora ocorreram entre 17 e 18 horas, com um total médio precipitado de $68 \mathrm{~mm}$. Estudos realizados por Costa et al. (2006a) também evidenciaram estas características das precipitações na FLONA de Caxiuanã. Em termos médios horários, durante os períodos chuvoso e seco, respectivamente, estes valores foram de 9,9 e 1,9 mm.

Os maiores valores médios horários da velocidade do vento ocorreram entre 10 e 13 horas, com máximo aquecimento diferencial da superfície, o que gera maiores gradientes de pressões atmosféricas e, consequentemente, maiores velocidades do vento. No horário entre 14 e 18 horas, estas velocidades médias oscilaram entre 10 e 1,4 m.s.-1. As menores velocidades médias horárias ocorreram entre 18 e 8 horas, apresentando valores inferiores a $0,5 \mathrm{~m} \cdot \mathrm{s}^{-1}$, o que está associado principalmente às menores diferenças térmicas na superfície, uma vez que, em grande parte destes intervalos de tempo, a superfície não recebe radiação solar, principal fonte de aquecimento dela. Em termos médios, durante os períodos chuvoso e seco, as velocidades do vento foram de 0,73 e $0,78 \mathrm{~m} \cdot \mathrm{s}^{-1}$, respectivamente.

\section{VARIABILIDADES MENSAL E HORÁRIA DA DIREÇÃO DO VENTO}

Nas Figuras 3 e 4, há, respectivamente, as variabilidades sazonal e horária da direção predominante do vento. Observou-se que durante grande parte do ano a direção do vento oscilou entre $45^{\circ}$ e $100^{\circ}$ (Nordeste-Leste). Ressalta-se que, durante o período menos chuvoso, esta predominância foi de Nordeste, fato este associado aos sistemas de grande escala atuantes na região (ventos alísios de Nordeste), enquanto no período chuvoso esta predominância foi de Leste. A predominância média anual da direção do vento foi de $107^{\circ}$ (E-SE), com desvio padrão de $11,2^{\circ}$ e coeficiente de variação de 10,4\%. Esta variabilidade mensal está diretamente associada aos sistemas produtores de precipitações que atuam naquela região, além dos efeitos de proximidade de corpos d'água, que geram oscilações neste elemento meteorológico.

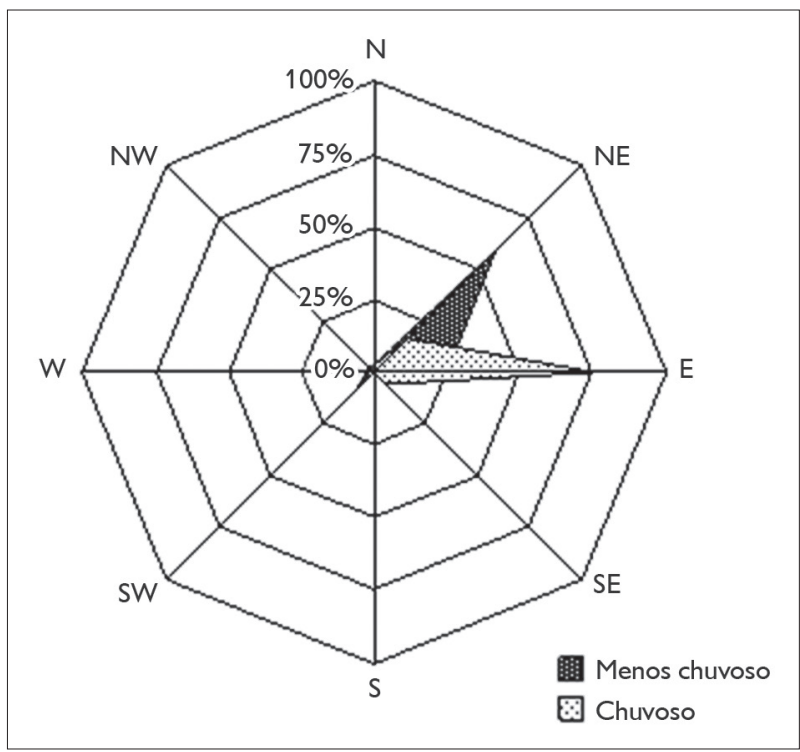

Figura 3. Variabilidade média anual da direção do vento na FLONA de Caxiuanã, no período de 2005 a 2013.

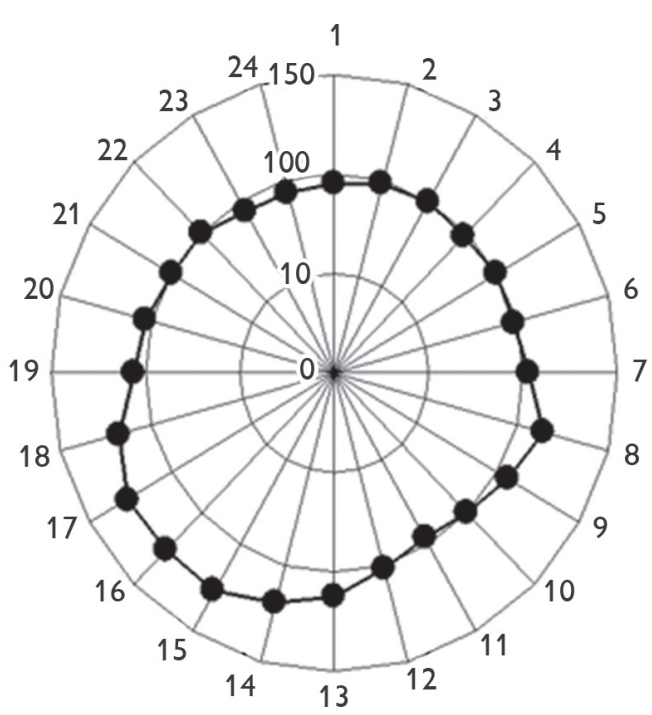

Figura 4. Variabilidade média horária da direção do vento na FLONA de Caxiuanã, no período de 2005 a 2013. 
Estes resultados estão de acordo com estudos realizados na FLONA de Caxiuanã por Braga et al. (2005).

Em relação à variabilidade média horária da direção do vento, observou-se que, durante grande parte do dia, entre 7 e 22 horas, o vento apresentou direção predominante de sudeste, tendo maiores tendências na direção Sul entre 13 e 18 horas. Estes desvios podem estar associados aos efeitos de brisa, relacionados com a proximidade da baía de Caxiuanã. No restante das horas do dia, esta predominância foi no quadrante Leste-Sudeste, associado aos efeitos da circulação atmosférica de grande escala atuantes na região, principalmente os ventos alísios de NE, corroborando os resultados dos estudos realizados por Camarinha Neto et al. (2011).

Em relação à análise circular (Ayres et al., 2005) para avaliação da variação sazonal ou mensal dos elementos meteorológicas, o valor- $p(p=0,1750)$ aponta para uma diferença não significativa entre as médias circulares dos três grupos. Assim, aceita-se a hipótese nula $\left(\mathrm{H}_{\mathrm{o}}\right)$ e conclui-se que nem a temperatura do ar nem a precipitação influenciam o padrão de direção do vento. Esta direção é representada pela média angular para esta amostra, com valor igual a 0,7083 (Leste para Oeste).

Entretanto, observou-se que o valor-p obtido para avaliar o sincronismo entre a direção do vento e a temperatura do ar resultou em nível de significância ( $p$ $=0,9455)$ e de intensidade do vento $(p=0,9955)$. A hipótese nula $\left(\mathrm{H}_{\mathrm{o}}\right)$ foi aceita, concluindo-se que, de fato, há sincronização entre a direção do vento e a sua intensidade, bem como com a temperatura do ar, na FLONA de Caxiuanã. Do mesmo modo, concluiu-se que também há, de fato, uma sincronização $(p=0,2013)$ entre a direção do vento e a precipitação. De modo contrário, concluiu-se que não há sincronização entre a direção do vento, a umidade relativa do ar e a radiação solar global ( $p<0,00001)$.

No segundo caso, o teste de Mann-Whitney (duas amostras relacionadas) foi utilizado para comparar todas as variações horárias para ambos os períodos sazonais (chuvoso e menos chuvoso). Em relação à temperatura do ar horária, o teste não foi significativo no limite da significância ( $p=0,0526)$, mas também é possível considerar este resultado como significativo. Do mesmo modo, em relação à umidade relativa do ar horária, o teste foi significativo ( $p=0,0335)$, assim como a precipitação $(p=0,0001)$ e a direção do vento $(p=0,0001)$. No entanto, não foi significativo em relação à radiação solar global $(0,6207)$ e à velocidade do vento $(p=0,5568)$.

\section{CONCLUSÕES}

Com base nas análises dos principais elementos meteorológicos na FLONA de Caxiuanã, nos anos de 2005 a 2013, considerando tanto as escalas horárias quanto as mensais, podemos concluir que a temperatura e a umidade relativa do ar apresentaram pouca variabilidade mensal durante todo o ano, entretanto sazonalmente com diferenças significativas.

Também concluiu-se que a precipitação foi o elemento meteorológico que apresentou a maior variabilidade estatística mensal e horária, sendo muito bem caracterizada a sua sazonalidade, um dos principais fatores ecológicos de regulaçãa em ecossistemas florestais tropicais.

A radiação solar global apresentou forte variação sazonal mensal, embora, em termos horários, não apresente diferenças sazonais. As variações neste quesito estão diretamente relacionadas com a variabilidade anual da nebulosidade e das precipitações.

A velocidade do vento apresentou valores relativamente baixos durante todo o ano, não apresentando diferenças significativas na escala horária, embora tenha apresentado diferenças significativas sazonalmente.

A direção predominante do vento refletiu claramente a atuação dos sistemas de pequena e de grande escala atuantes na região. Com base nas análises estatísticas circulares, houve significativa associação entre este elemento meteorológico com a velocidade do vento e precipitação, não havendo este sincronismo com a temperatura do ar, a umidade relativa do ar e a radiação solar global. 
Ressalta-se, ainda, a influência meteorológica local, principalmente associada com a proximidade da baía de Caxiuanã, que pode interferir na variação da direção do vento local, associado com o efeito de brisa, em função da grande massa líquida localizada nas proximidades da área de monitoramento.

\section{AGRADECIMENTOS}

Os autores agradecem ao Museu Paraense Emílio Goeldi (MPEG), ao Conselho Nacional de Desenvolvimento Científico e Tecnológico (CNPq) e a todas as pessoas envolvidas de forma direta e indireta com as atividades do Projeto Seca Floresta (ESECAFLOR).

\section{REFERÊNCIAS}

ALMEIDA, S. S., P. L. B. LISBOA \& A. S. L. SILVA, 1993. Diversidade florística de uma comunidade arbórea na Estação Científica "Ferreira Penna", em Caxiuanã (Pará). Boletim do Museu Paraense Emílio Goeldi, série Botânica 9(1): 93-128.

AYRES, M., M. AYRES JR., D. L. AYRES \& A. S. SANTOS, 2005. Bioestat 5.0: aplicações nas áreas de ciências biológicas e médicas. IDSM/MCT/CNPq, Tefé.

BRAGA, A. P., J. A. SILVA JÚNIOR, A. C. L. COSTA, P. H. L. GONÇALVES, R. F. COSTA, R. B. PACHECO, N. E. F. NÓBREGA, P. MEIR \& Y. MALHI, 2005. Comportamento sazonal de alguns elementos meteorológicos na Floresta Nacional de Caxiuanã Pará - Brasil. Anais do Congresso Latinoamericano e Ibérico de Meteorologia/Congresso Cubano de Meteorologia 10/2: 1 CD-ROM.

CAMARINHA NETO, G. F., A. C. L. COSTA, A. A. R. OLIVEIRA \& M. C. COSTA, 2011. Perfil trimestral, mensal e horário da velocidade e direção do vento às margens da baía de Caxiuanã, Melgaço, PA: estudo de caso. Anais do Congresso Brasileiro de Agrometeorologia 17: 1-5.

COSTA, A. C. L., A. P. BRAGA, P. H. L. GONCALVES, R. F. COSTA, J. A. SILVA JÚNIOR, Y. MALHI, L. ARAGÃO \& P. MEIR, 2006a. Estudos hidrometeorológicos em uma floresta tropical chuvosa na Amazônia. Revista Brasileira de Meteorologia 21(3b): 283-290.

COSTA, A. C. L., P. H. L. GONÇALVES, A. P. BRAGA, J. A. SILVA JÚNIOR \& M. C. COSTA, 2006b. Sazonalidade da radiação solar global, direta e difusa em uma floresta tropical chuvosa na Amazônia oriental - FLONA Caxiuanã - Pará. Anais do Congresso Brasileiro de Meteorologia 14: 1 CD-ROM.
COSTA, A. C. L., S. S. ALMEIDA, C. CARVALHO, P. MEIR, Y. MALHI, R. F. S. J. COSTA, J. ATHAYDES, M. C. COSTA, B. T. T. PORTELA, R. A. FISHER, E. D. SOTTA, L. L. OLIVEIRA, P. H. GONÇALVES, A. P. BRAGA, P. N. BARRETO, 2009. Experimento ESECAFLOR-LBA em Caxiuanã. In: P. L. B. LISBOA (Org.): Caxiuanã: desafios para a conservação de uma Floresta Nacional na Amazônia: v. 1: p. 45-91. MPEG, Belém.

COSTA, A. C. L., D. GALBRAITH, S. ALMEIDA, B. T. T. PORTELA, M. DA COSTA, J. A. SILVA JUNIOR, A. P. BRAGA, P. H. L. GONÇALVES, A. A. R. OLIVEIRA, R. FISHER, O. L. PHILLIPS, D. B. METCALFE, P. LEVY \& P. MEIR, 2010. Effect of 7 yr of experimental drought on vegetation dynamics and biomass storage of an eastern Amazonian rainforest. New Phytologist 187(3): 579-591. DOI: http://dx.doi.org/10.1111/j.1469-8137.2010.03309.x.

DOUGHTY, C. E., D. B. METCALFE, C. A. J. GIRARDIN, F. F. AMÉZQUITA, D. G. CABRERA, W. HUARACA HUASCO, J. E. SILVA-ESPEJO, A. ARAUJO-MURAKAMI, M. C. COSTA, W. ROCHA, T. R. FELDPAUSCH, A. L. M. MENDOZA, A. C. L. COSTA, P. MEIR, O. L. PHILLIPS \& Y. MALHI, 2015. Drought impact on forest carbon dynamics and fluxes in Amazonia. Nature 519: 78-82. DOI: http://dx.doi.org/10.1038/nature14213.

FISCH, G., J. A. MARENGO \& C. A. NOBRE, 1996. Clima da Amazônia. Climanálise - Boletim de Monitoramento e Análise Climática - Ed. Comemorativa de 10 anos, cap. 3, [s.n], Cachoeira Paulista, n.p.

FISCH, G. F., J. A. MARENGO \& C. A. NOBRE, 1998. Uma revisão geral do clima da Amazônia. Acta Amazonica 28(2): 101-126. DOI: http://dx.doi.org/10.1590/1809-43921998282126.

GAT, J. R. \& E. MATSUI, 1991. Atmospheric water balance in the Amazon Basin: an isotopic evapotranspiration model. Journal of Geophysical Research 96(D7): 13179-13188. DOI: http://dx.doi. org/10.1029/91JD00054.

LISBOA, P. L. B., A. S. L. SILVA \& S. S. ALMEIDA, 1997. Florística e estrutura dos ambientes. In: P. L. B. LISBOA (Org.): Caxiuanã: 163-193. Museu Paraense Emílio Goeldi, Belém.

MARENGO, J. C. \& J. A. ESPINOZA, 2016. Review: Extreme seasonal droughts and floods in Amazonia: causes, trends and impacts. International Jounal of Climatology 36(3): 1033-1050. DOI: http://dx.doi.org/10.1002/joc.4420.

MOLION, L. C. B., 1987. On the dynamic climatology of the Amazon basin and associated rain-producing mechanisms. In: R. E. DICKINSON (Ed.): The Geophysiology of Amazonia vegetation and climate interactions: 391-407. John Wiley and Sons, New York.

MORAES, J. C., J. P. R. COSTA, E. J. P. ROCHA \& I. M. O. SILVA, 1997. Estudos hidrometeorológicos na bacia do rio Caxiuanã. In: P. L. B. LISBOA (Org.): Caxiuanã: 85-95. CNPq/Museu Paraense Emílio Goeldi, Belém. 
OLIVEIRA, L. L., R. F. COSTA, F. A. S. SOUSA, A. C. L. COSTA \&A. P. BRAGA, 2008. Precipitação efetiva e interceptação em Caxiuanã, na Amazônia Oriental. Acta Amazonica 38(4): 723-732. DOI: http:// dx.doi.org/10.1590/S0044-59672008000400016.

ROWLAND, L., A. C. L. COSTA, D. R. GALBRAITH, R. S. OLIVEIRA, O. J. BINKS, A. A. R. OLIVEIRA, A. M. PULLEN, C. E. DOUGHTY, D. B. METCALFE, S. S. VASCONCELOS, L. V. FERREIRA, Y. MALHI, J. GRACE, M. MENCUCCINI \& P. MEIR, 2015. Death from drought in tropical forests is triggered by hydraulics not carbon starvation. Nature 528(7580): 119-122. DOI: http:// dx.doi.org/10.1038/nature15539.

SILVAJÚNIOR, J. A., A. C. L. COSTA, P. V. AZEVEDO, R. F. COSTA, D. B. METCALFE, P. H. L. GONÇALVES, A. P. BRAGA, Y. MALHI, L. E. O. C. ARAGÃO \& P. MEIR, 2013. Fluxos de $\mathrm{CO}_{2}$ do solo na Floresta Nacional de Caxiuanã, Pará, durante o experimento ESECAFLOR/LBA. Revista Brasileira de Meteorologia 28(1): 8594. DOI: http://dx.doi.org/10.1590/S0102-77862013000100009.
SOUZA, E. S., A. O. MANZI, A. C. CUNHA, G. SAMPAIO, L. A. CANDIDO, E. P. ROCHA, J. M. B. ALVES, M. F. CARDOSO, A. M. L. SOUSA \& M. M. COUTINHO, 2014. Mudanças ambientais de curto e longo prazo: projeções, reversibilidade e atribuição. In: T. AMBRIZZI \& M. ARAÚJO (Ed.): Base científica das mudanças climáticas: primeiro relatório de avaliação nacional: v. 1: 322-336. Painel Brasileiro de Mudanças Climáticas, Rio de Janeiro.

TABARELLI, M., C. F. D. ROCHA, H. P. ROMANOWSKI, O ROCHA \& L. D. LACERDA, 2013. PELD-CNPq: dez anos do Programa de Pesquisas Ecológicas de Longa Duração do Brasil: achados, lições e perspectivas: 1-446. Editora Universitária UFPE, Recife.

VILANI, M. T., L. SANCHES, J. S. NOGUEIRA \& N. PRIANTE FILHO, 2006. Sazonalidade da radiação, temperatura e umidade em uma floresta de transição Amazônia Cerrado. Revista Brasileira de Meteorologia 21(3b): 331-343. 
